\title{
Modelling energy consumption in test cells
}

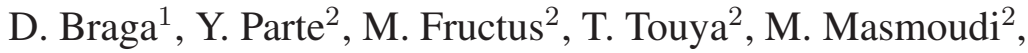 \\ T. Wylot ${ }^{3} \&$ V. Kearley ${ }^{3}$ \\ ${ }^{1}$ ACTIS, Avenue de Catalogne, 11300 Limoux, France \\ ${ }^{2}$ Institut de Mathématique de Toulouse, 31062 Toulouse, France \\ ${ }^{3}$ TRADA Technology Ltd, High Wycombe, HP144ND, U.K.
}

\begin{abstract}
In situ measurements of energy consumption in test cells are often carried out to predict the thermal performance of the insulating product. Design engineers need a tool that can use available in situ measurements to model the thermal performance of the test cell at any location world wide and also permit comparison of different insulation products. Towards this goal, we present GAP, Global Assimilation Process which is a neural network based meta modeling technique. The key feature of this method is the zero memory minimization routine and a regularization technique that avoids over training of the network. We present the theory and applications of GAP software to predict thermal performance of mineral wool and multi-foil insulation products. GAP based meta models are used to predict thermal performance of these insulation products at different test sites. It is shown that the properly trained neural network model of GAP can accurately predict the energy consumption in test cells of any location.
\end{abstract}

Keywords: In situ measurements, neural network, GAP, multifoil insulation.

\section{Introduction}

Thermal properties of insulation materials such as U-value or R-value are measured using controlled laboratory tests that employ simplistic specimen geometries and boundary conditions [1,2] or by established standards [3]. However, a detailed report from the Building Research Establishment Ltd. [4] shows the considerable differences amongst standard calculation method such as [3] and in situ measurements. The difference arises from the fact that the laboratory conditions or standards does not simulate factors such as wind, solar radiation, relative humidity, 
infiltration, etc., which influence in situ performance. Hygrothermal performance modeling softwares which claim to model these factors are inadequate as they employ simplifying assumptions for wind profiles and solar radiation. Realistic estimation of in situ performance of insulation products requires a tool that can couple the model and the measured data. Moreover, such model should be computationally inexpensive so that design engineers can use it to model the thermal performance of the test cell at any location world wide and also permit comparison of different insulation products. Towards this goal, we present Global Assimilation Process (GAP) which is a neural network based meta modeling technique. In section 2 we give a description of GAP, whose key features are use of algorithmic differentiation to develop a low-memory Levenberg-Marquardt algorithm and the use of a regularization technique; by which it provides a neural networks that do not "over fit" the given data. Using GAP, the neural network is trained to predict in situ consumption data corresponding to various meteorological conditions. The in situ data collection strategy and test set up are discussed in section 3. Finally in section 4 it is shown that properly trained neural network can accurately predict the energy consumption in test cells located at other geographic locations.

\section{GAP theory}

The core idea of GAP is to build neural network based model of the energy consumption in test cells as a function of meteorological parameters. The model parameters are tuned by minimizing the difference between the measured and simulated consumption. We assume that energy consumption is a continuous function $\psi$ defined from $\mathbb{R}^{n_{I}}$ ( $n_{I}$ input variables, for e.g. meteorological parameters) into $\mathbb{R}^{n_{O}}$ ( $n_{O}$ output variables, for e.g. energy consumption). In following subsections we describe neural network model and the training algorithm.

\subsection{Neural networks}

We consider three-layer neural networks, as they are universal approximators for continuous functions [5-7]. The first layer is the input layer and contains $n_{I}+1$ cells corresponding to the $n_{I}$ input variables, and an additional cell which is called the bias. The second (or intermediate) layer is called the hidden layer, consists of $n_{H}$ hidden cells, $n_{H}$ usually being increased with the complexity of the function to be approximated. The third layer is the output layer and contains $n_{O}$ cells. Each cell $c_{j}$ of one layer is connected to each cell $c_{i}$ of the following layer, and each of these links is associated to a weight $w_{i j}$. If we denote by $x_{i}^{l}$ the state of cell $c_{i}$ of the layer $l$, then the state of cell $c_{j}$ of the second layer is given by

$$
x_{j}^{2}=f\left(\left(\sum_{k=1}^{n_{I}} w_{j k}^{1} x_{k}^{1}\right)+w_{j, n_{I}+1}^{1}\right)
$$

where $f$ is the activation function given by eqn. (2).

$$
f(z)=\frac{1}{1+e^{-z / 10}} \forall z \in \mathbb{R}
$$


This means that some basis functions are built between the input and hidden layers. The state of cell $c_{i}$ of the output layer is finally given by a linear approximation in this basis

$$
x_{i}^{3}=\sum_{j=1}^{n_{H}} w_{i j}^{2} x_{j}^{2}
$$

We will now consider the following vectorial notations:

- $X^{1}=\left(x_{1}^{1}, \ldots, x_{n_{I}}^{1}\right)^{T}$ is the input vector, $\underline{X^{1}}=\left(x_{1}^{1}, \ldots, x_{n_{I}}^{1}, 1\right)^{T}$, and $X^{3}=\left(x_{1}^{3}, \ldots, x_{n_{O}}^{3}\right)^{T}$ is the output vector.

- $W^{1}$ is the $n_{H} \times\left(n_{I}+1\right)$ matrix formed with the weights $w_{j k}^{1}$, and $W^{2}$ is the $n_{O} \times n_{H}$ matrix formed with the $w_{i j}^{2} . W=\left(W^{1}, W^{2}\right) \in \mathbb{R}^{n_{H} \times\left(n_{I}+1\right)} \times$ $\mathbb{R}^{n_{O} \times n_{H}}$.

- $F$ is the function defined for all $X^{2}=\left(x_{1}^{2}, \ldots, x_{n_{H}}^{2}\right)^{T}$ by $F\left(X^{2}\right)=$ $\left(f\left(x_{1}^{2}\right)\right.$, $\left.\ldots, f\left(x_{n_{H}}^{2}\right)\right)^{T}$

With these notations, the response $R$ of the neural network to the input $X^{1}$ with the weights $W$ is simply given by

$$
X^{3}=R\left(W, X^{1}\right):=W^{2} F\left(W^{1} \underline{X^{1}}\right)
$$

\subsection{Training of neural networks}

Consider the observation set $\Omega$ with $n_{P}$ observations

$$
\Omega=\left\{\left(X_{i}, Y_{i}\right), i=1, \ldots, n_{P}\right\}
$$

in which each $X_{i} \in \mathbb{R}^{n_{I}}$ is a vector corresponding to input variables, and $Y_{i} \in$ $\mathbb{R}^{n_{O}}$ is the response to the input $X_{i}$. For each observation we denote by $r_{i}(W)=$ $R\left(W, X_{i}\right)-Y_{i}$ the residual and $r(W)=\left(r_{1}(W), \ldots, r_{n_{p}}(W)\right)^{T}$ will be called residual vector. The difference between neural network output and observed response is called the discrepancy function and is given by eqn. (6).

$$
G_{\Omega}(W)=\left(r_{1}, \ldots, r_{n_{P}}\right) \in \mathbb{R}^{n_{O} \times n_{P}}
$$

In order to make the neural network a good approximation model, we minimize the difference between the network output and the observed response, i.e. we look for the weights $\hat{W}$ which is a solution of the following minimization problem

$$
\min _{W} h_{\Omega}(W):=\frac{1}{2}\left\|G_{\Omega}(W)\right\|^{2}
$$

The minimization problem is solved using zero memory Levenberg-Marquardt algorithm as described in section 2.3. First, the set of patterns $\Omega$ is divided into three parts, the training set $\Omega_{T}$, the generalization set $\Omega_{G}$, and the validation set $\Omega_{V}$. The initial weights $W_{0}$ are set to small random values between -0.1 and 0.1 and the neural network is trained in following three phases. 


\subsubsection{First training phase}

We look for the size of the hidden layer $n_{H}$ that allows us to make a good training of the neural network on the patterns from $\Omega_{T}$. This means that $n_{H}$ is automatically increased, and

$$
h_{\Omega_{T}}\left(\hat{W}_{0}\right)=\min _{W} h_{\Omega_{T}}(W)
$$

is solved until $h_{\Omega_{T}}\left(\hat{W}_{0}\right)$ becomes smaller than a threshold precision $\eta>0$.

\subsubsection{Regularization phase}

We now add a Tikhonov regularization term to the functional $h_{\Omega_{T}}$ and we look for the regularization parameter $\beta$ in order to enforce the weights involved in the definition of the basis functions to remain small. By this way, we try to define smooth and stretched basis functions that will prevent the neural network to oscillate too much.

- If $h_{\Omega_{G}}\left(\hat{W}_{0}\right)<\eta$, then we set $\hat{\beta}=0$;

- otherwise, for several increasing values of $\beta>0$, we look for $\hat{W}_{\beta}$ that solves

$$
\min _{W}\left[h_{\Omega_{T}}(W)+\beta \frac{1}{2\left(n_{I}+1\right) n_{H}}\left\|W^{1}\right\|^{2}\right]
$$

In this minimization problem weights are initialized to the value obtained at the end of first training phase. We denote by $\hat{\beta}$ the value of $\beta$ for which $h_{\Omega_{G}}\left(\hat{W}_{\beta}\right)$ is the smallest.

\subsubsection{Final training phase}

We perform finally a new training phase on the set $\Omega_{T} \cup \Omega_{G}$ using the regularization parameter $\hat{\beta}$ provided by the previous step and $\hat{W}_{\beta}$ as initial weights.

$$
\min _{W}\left[h_{\Omega_{T} \cup \Omega_{G}}(W)+\hat{\beta} \frac{1}{2\left(n_{I}+1\right) n_{H}}\left\|W^{1}\right\|^{2}\right]
$$

\subsection{Levenberg-Marquardt algorithm}

Levenberg-Marquardt algorithm is a combination of steepest descent method and Gauss-Newton algorithm [8-10]. The iterative descent algorithms consist in defining a descent direction $d$, and the new point $W_{+}$is obtained from the current point $W$ using the following update rule

$$
W_{+}=W+d
$$

The descent direction, $d$ is given by [10]

$$
\left(J(W)^{T} J(W)+\alpha I\right) d=-J(W)^{T} r(W)
$$

where $\alpha>0$ and $J(W)=\nabla r(W)^{T}$ is the Jacobian matrix. The LevenbergMarquardt algorithm is then the following:

- Choose an initial point $W_{0}$ and a real number $\alpha_{0}>0, k=0$ 
- Compute $d_{k}$, solution to $\left(J\left(W_{k}\right)^{T} J\left(W_{k}\right)+\alpha_{k} I\right) d_{k}=-J\left(W_{k}\right)^{T} r\left(W_{k}\right)$

- If $h_{\Omega}\left(W_{k}+d_{k}\right)<h_{\Omega}\left(W_{k}\right)$, set $W_{k+1}=W_{k}+d_{k}$, choose $\alpha_{k+1}<\alpha_{k}$, increase $k$ and goto previous step; otherwise, decrease $\alpha_{k}$ and goto previous step. Stop if $h_{\Omega}\left(W_{k}+d_{k}\right)<\eta$

\subsubsection{Memory reduction and adjoint computation}

As seen in eqn. (12), the Levenberg-Marquardt algorithm usually requires the computation of the inverse of $J(W)^{T} J(W)+\alpha I$, whose size may be quite large in some cases. For memory reduction, at least in terms of storage, the linear system in eqn. (12) can be solved using the conjugate gradient method, which requires only matrix-vector products. We only need to compute the left-hand side of eqn.(12) in an efficient way. This can be done in two steps.

1 . We first compute $z=J(W) d$. This quantity can be rewritten as follows

$$
J(W) d=\lim _{\varepsilon \rightarrow 0} \frac{r(W+\varepsilon d)-r(W)}{\varepsilon}=\left.\frac{\partial r(W+\varepsilon d)}{\partial \varepsilon}\right|_{\varepsilon=0}
$$

and $J(W) d$ corresponds to the differentiation of a vector-valued function $r$ with respect to a single parameter $\varepsilon$. This can be done very efficiently using the forward mode of the algorithmic differentiation.

2. Then, we have to compute $J(W)^{T} z$, which can be rewritten

$$
J(W)^{T} z=\sum_{i=1}^{n_{P}} \nabla r_{i}(W) z_{i}=\nabla\left(\sum_{i=1}^{n_{P}} r_{i}(W) z_{i}\right)=\nabla\left(r(W)^{T} z\right)
$$

In this form, $J(W)^{T} z$ corresponds to the differentiation of a scalar function with respect to several parameters and the reverse mode of the algorithmic differentiation is particularly efficient in this case [11-13].

The computation of the right-hand side of (12) is realized in the same manner as in the second step of the computation of the left-hand side.

\section{Experimental setup}

\subsection{Description of the test cells}

Two test cells, located at Limoux, France, with a roof surface area of $35 \mathrm{~m}^{2}$, outside dimensions of $4 \times 7 \mathrm{~m}^{2}$ on floor level and height of $3 \mathrm{~m}$ were used for the test. Each cell is built without windows and there is no ventilation. The roof with inclination of $36^{\circ}$ is made up of rafter of $8 \times 11 \mathrm{~cm}$ with spacing of $48 \mathrm{~cm}$ between adjacent rafters and has clay tiles. The roof ridge has north-south orientation. The floor is made up of wood paving and the under floor gap is over insulated with $40 \mathrm{~cm}$ of mineral wool. The access to each test volume is by an airlock in the gable wall and thus the thermal exchange takes place through walls and roof alone. Inside temperature of each cell is maintained at $23^{\circ} \mathrm{C}$ using two fan heater of $1 \mathrm{KW}$ output. The airlock is heated to $1^{\circ} \mathrm{C}$ less than the main cell and acts as a guard cell. One test 
cell has TS9 multi-foil insulation of ACTIS [14] and other has $20 \mathrm{~cm}$ of mineral wool insulation. The layout of each cell is in accordance with the manufacturer's instructions [14]. Infra red pictures made outside and inside of the test cells do not reveal any significant differences between each kind of cells.

\subsection{Instrumentation and measurements}

Each cell is equipped with two temperature sensors located $1.5 \mathrm{~m}$ above the floor and placed in an open gray PVC tube to shield them from air movements. Energy consumption in each test cell is measured by recording current and voltage using calibrated instruments. Weather parameters namely, the outside temperature, relative humidity, wind direction, wind speed and total solar radiation were recorded per minute by a dedicated weather station at the site. The period of measurement was from 1 December 2005 to 28 February 2006. Measurements are carried out per minute and are recorded by dedicated data logger units. All quality control checks pertaining to instrumentations were made. [14]

\section{Simulations using GAP}

\subsection{Training of neural network}

The consumption in a test cell is a function of meteorological parameters, namely, the difference between outside temperature and temperature inside the chalet, wind speed, wind direction, relative humidity and global solar radiation. Using GAP, two neural networks pertaining to the data of two test cells are developed. Total 1513 observations spanned over the period $1 / 12 / 2005$ to $31 / 12 / 2005$ are used to train the network. These networks are validated by predicting the consumption values for the period of 1/1/2006 to 28/02/2006. Fig. 1, 2 shows the comparison between measured and simulated energy consumptions corresponding to these experiments.

Table 1 shows the measured and simulated energy consumption in these test cells. The difference between measured and simulated consumption is less than $1 \%$. It is observed that the energy consumption in a test cell with TS9 material is $4 \%$ less than the energy consumption for a test cell with mineral wool.

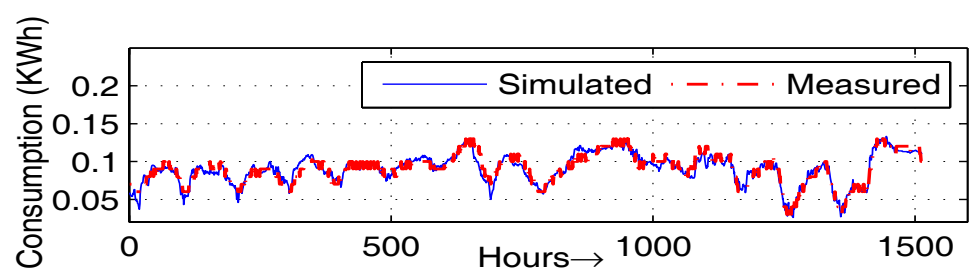

Figure 1: Comparison of measured and simulated energy consumption in a test cell with TS9 insulation, located at Limoux, France. 


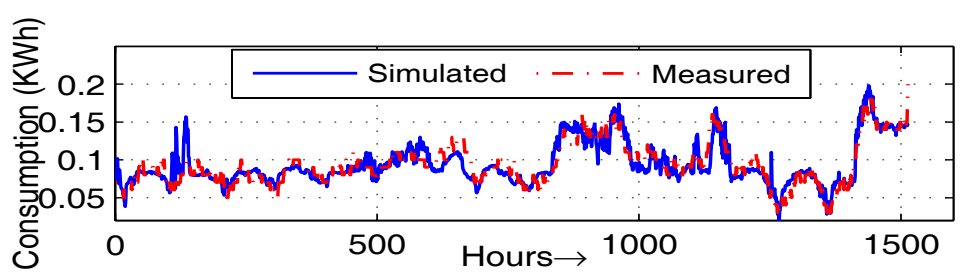

Figure 2: Comparison of measured and simulated energy consumption in a test cell with mineral wool insulation, located at Limoux, France.

Table 1: Measured and simulated net energy consumption in test cells located at Limoux, France.

\begin{tabular}{|c|c||c|c|}
\hline \multicolumn{4}{|c|}{ Energy consumption in kWh } \\
\hline \multicolumn{2}{|c||}{ Cell with TS9 } & \multicolumn{2}{c|}{ Cell with mineral wool } \\
\hline Measured & Simulated & Measured & Simulated \\
\hline 137 & 136 & 143 & 142 \\
\hline
\end{tabular}

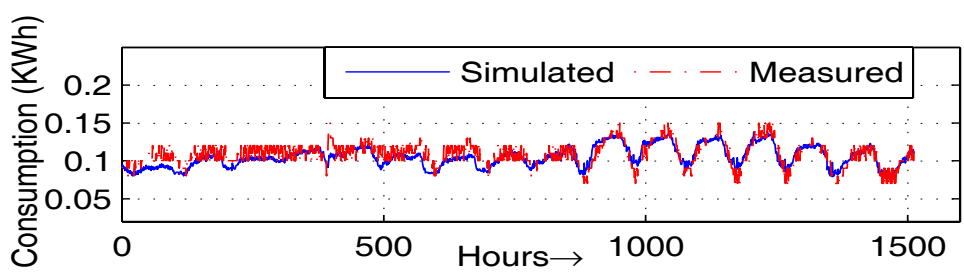

Figure 3: Comparison of measured and simulated energy consumption in a test cell with multi-foil insulation, located at TRADA, U.K.

\subsection{Prediction using neural network}

These trained and validated neural networks are used to predict the energy consumption of similar test cells with multi-foil insulation similar to TS9 and mineral wool insulation located at TRADA in United Kingdom [15]. The weather data and the consumption were measured for the period 1/1/2006 to 28/2/2006. Fig. 3, 4 shows the comparison of measured and simulated consumption. Table 2 provides the values of measured and simulated consumption. For the cell with multi-foil insulation the simulated consumption differs by $4 \%$ where as for the test cell with mineral wool insulation the difference is $3 \%$.

As part of the long term in situ data collection strategy, ACTIS planned to set up new test cells in United Kingdom. The weather data spanned over the 


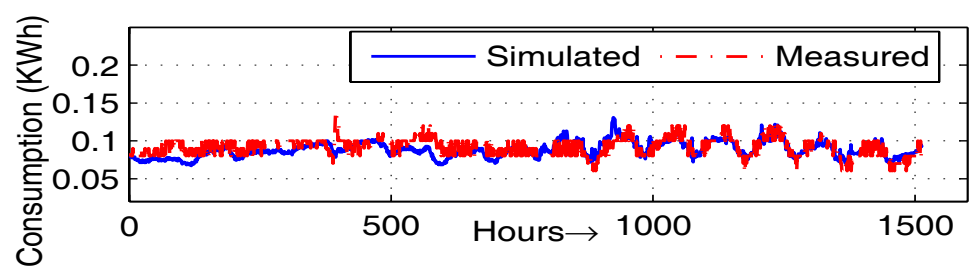

Figure 4: Comparison of measured and simulated energy consumption in a test cell with mineral wool insulation, located at TRADA, U.K.

Table 2: Measured and simulated net energy consumption in test cells at TRADA, United Kingdom.

\begin{tabular}{|c|c||c|c|}
\hline \multicolumn{4}{|c|}{ Energy consumption in $\mathrm{kWh}$} \\
\hline Cell with multi-foil insulation & Cell with mineral wool \\
\hline Measured & Simulated & Measured & Simulated \\
\hline 165 & 159 & 138 & 134 \\
\hline
\end{tabular}

period of 1/2/2006 to 28/02/2006 at eight different location in United Kingdom, namely, Manchester, Norwich, London, Plymouth, Cardiff, Aberdeen, Newcastle and Belfast is available. The weather data characteristics at these locations are different than those at Limoux, France. Fig. 5 shows the range of measured values for outside temperature, wind direction, wind speed and global solar radiation at Limoux, France and at these location in United Kingdom. The minimum temperature at these locations is higher than Limoux whereas global solar radiation values are small compared to Limoux. In order to estimate the energy consumption in test cells that would be built in near future at these sites, the neural network model trained using weather data and consumption details in Limoux, France is used to simulate the in situ energy consumption in test cells with TS9 and mineral wool insulation materials.

Table 3 shows predicted energy consumption for the test cell with TS9 and mineral wool insulation to be built at each of the eight sites. The difference in energy consumption for the test cell with TS9 and mineral wool insulations is also shown. The predicted consumption in test cell with multi-foil insulation is less compared to test cell with mineral wool insulation at Plymouth, Cardiff, Aberdeen, Newcastle and Belfast whereas it is more by $2 \%$ at Manchester and by $1 \%$ at Norwich and London. The standard deviation of predicted consumption values for test cell with mineral wool is 9.7 whereas the corresponding values for the test cell with multifoil insulation is 6.5 , possibly indicating that multifoil insulation is more robust to varying weather conditions as compared to mineral wool insulation. 


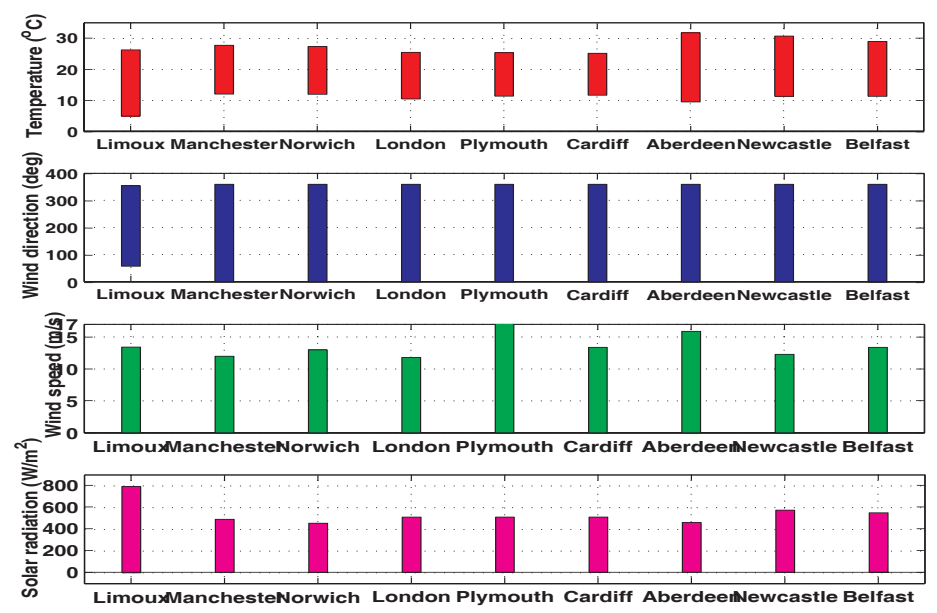

Figure 5: Comparison of weather data at eight different locations in U.K. and of Limoux, France.

Table 3: Simulated consumptions of test cells with ACTIS TS9 and mineral wool insulation at eight different locations in United Kingdom.

\begin{tabular}{|l|c|c|c|}
\hline \multirow{2}{*}{ Location } & \multicolumn{2}{|c|}{ Energy consumption (kWh) } & Difference \\
\cline { 2 - 3 } & $\begin{array}{c}\text { Mineral wool } \\
(\mathrm{A})\end{array}$ & $\begin{array}{c}\text { ACTIS TS9 } \\
(\mathrm{B})\end{array}$ & \\
\hline Manchester & 135 & 138 & $2 \%$ \\
\hline Norwich & 136 & 137 & $1 \%$ \\
\hline London & 129 & 130 & $1 \%$ \\
\hline Plymouth & 155 & 128 & $-17 \%$ \\
\hline Cardiff & 132 & 126 & $-5 \%$ \\
\hline Aberdeen & 154 & 143 & $-7 \%$ \\
\hline Newcastle & 144 & 143 & $-1 \%$ \\
\hline Belfast & 140 & 136 & $-3 \%$ \\
\hline
\end{tabular}

\section{Conclusion}

GAP is a result of a powerful combination of several techniques such as the use of a zero memory minimization method, specific activation function that guarantees 
the minimal change of weights, the use of a Tikhonov regularization technique in order to build smooth and stretched basis functions. GAP is used to generate neural network model to predict energy consumption of test cells as a function of meteorological parameters using in situ data. It is demonstrated with examples that properly trained networks can accurately predict the energy consumption of houses located at some other locations also.

\section{References}

[1] ISO 8302, Thermal insulation. Determination of steady state thermal resistance and related properties-Gaurded hot plate apparatus.

[2] ISO 8990:1994, Thermal insulation. Determination of steady state thermal transmission properties-Calibrated and guarded hot box.

[3] ISO 6946, Building components and building elements-Thermal resistance and thermal transmittance-Calculation method.

[4] Doran, S., Field investigation of thermal performance of construction elements as built. Technical Report Building Research Establishment Ltd. 78132, November, 2001.

[5] Cybenko, G., Continuous valued neural networks with two hidden layers are sufficient. Technical report, Department of Computer Science,Tufts University, Mdeford, Massachusetts, 1988.

[6] Cybenko, G., Approximation by superpositions of sigmoidal function. Mathematics of control, signals and systems, 2, pp. 303-314, 1989.

[7] K. Hornik, M.S. \& White, H., Multilayer feedforward networks are universal approximators. Neural networks, 2, pp. 359-366, 1989.

[8] Levenberg, K., A method for the solution of certain problems in least squares. Quarterly of Applied Mathematics, 2, pp. 164-168, 1944.

[9] Marquardt, D., An algorithm for least-squares estimation of nonlinear parameters. SIAM Journal on Applied Mathematics, 11, pp. 431-441, 1963.

[10] Nocedal, J. \& Wright, S., Numerical Optimization. Springer Series in Operations Research and Financial Engineering, Springer, 2nd edition, 2006.

[11] J. Gilbert, G.V. \& Masse, J., La differentiation automatique de fonctions representes par des programmes. Technical Report 1557, Technical report,INRIA, 1991.

[12] Griewank, A., Evaluating derivatives: principles and techniques of algorithmic differentiation. SIAM: Philadelphia, USA, 2000.

[13] Rall, L.B. \& Corliss, G.F., An introduction to automatic differentiation. Computational Differentiation: Techniques, Applications, and Tools, eds. M. Berz, C.H. Bischof, G.F. Corliss \& A. Griewank, SIAM: Philadelphia, USA, pp. 1-17, 1996.

[14] ACTIS. http://www.actis-isolation.com.

[15] Kearley, V., Multifoil testing at TRADA. Technical report, TRADA Technology Ltd, United Kingdom. 